\title{
Finite-element simulations of the influence of pore wall adsorption on cyclic voltammetry of ion transfer across a liquid-liquid interface formed at a micropore
}

\author{
Jonathan S. Ellis, ${ }^{a *}$ Jörg Strutwolf, ${ }^{a \llbracket l}$ Damien W. M. Arrigan ${ }^{b}$ \\ 5
}

Adsorption onto the walls of micropores was explored by computational simulations involving cyclic voltammetry of ion transfer across an interface between aqueous and organic phases located at the micropore. Micro-interfaces between two immiscible electrolyte solutions (microITIES) have been of particular research interest in recent years and show promise for biosensor and biomedical applications. The simulation model combines diffusion to and within the micropore, Butler-Volmer kinetics for ion transfer at the liquid-liquid interface, 10 and Langmuir-style adsorption on the pore wall. Effects due to pore radius, adsorption and desorption rates, surface adsorption site density, and scan rate were examined. It was found that the magnitude of the reverse peak current decreased due to adsorption of the transferring ion on the pore wall; this decrease was more marked as the scan rate was increased. There was also a shift in the half-wave potential to lower values following adsorption, consistent with a wall adsorption process which provides a further driving force to transfer ions across the ITIES. Of particular interest was the disappearance of the reverse peak from the cyclic voltammogram at higher 15 scan rates, compared to the increase in the reverse peak size in the absence of wall adsorption. This occurred for scan rates of $50 \mathrm{mV} / \mathrm{s}$ and above and may be useful in biosensor applications using micropore-based ITIES.

\section{Introduction}

Liquid|liquid interfaces have received recent attention as tools for 20 label-free detection of various ionic species in a wide range of media. ${ }^{1-3}$ Such interfaces between two immiscible electrolyte solutions (ITIES) have been used to study ion transfer processes involving organic and inorganic species, ${ }^{4,5}$ including ions of biological interest. ${ }^{6}$ Facilitated ion transfer has also been 25 explored, whereby ionophores in the organic phase ${ }^{7,8}$ or biological macromolecules ${ }^{9,10}$ adsorbed at the interface enable the transfer of species that otherwise would not transfer. This concept has been investigated recently to examine a possible time-dependent conformational shift of the protein hemoglobin 30 adsorbed at a water|1,2-dichloroethane interface. ${ }^{11}$ The main analytical advantage of electrochemistry at the ITIES is that ionic species can be detected directly in a label-free manner. Additionally, devices formed from such ITIES show promise as tools for exploring biological-like processes involving the 35 transfer of ions.

Recently, micropores ${ }^{12-15}$ and nanopores ${ }^{7,16-19}$ have been used to form miniaturized ITIES, so as to improve mass transfer fluxes and increase current densities. Mass transfer to millimetre-sized ITIES, as to similarly-sized electrodes, occurs via linear ${ }_{40}$ diffusion. In this regime, diffusion of ions occurs in one dimension towards the interface. This is reflected in current peaks in the voltammograms, followed by a decay of the current proportional to the square root of time. For micro- and nanoITIES, edge effects become important, wherein radial 45 diffusion occurs around the ITIES in the time frame of electrochemical experiments. This serves to increase the areal mass transfer flux, and thus the ion current density, with followon effects on parameters of analytical importance such as the limit-of-detection, signal-to-noise ratio and sensitivity. The 50 resulting cyclic voltammetry $(\mathrm{CV})$ plot tends towards a steadystate, sigmoidal shape. This is even the case for arrays of microITIES, provided there is sufficient separation between the adjacent micropores used to form the micro-interfaces. ${ }^{14,15,20}$ or if the overall size of the array is in the $\mu \mathrm{m}$ range. ${ }^{21}$ This is
55 analogous to similar behaviour predicted for arrays of microelectrodes. $^{22-25}$

ITIES and microITIES have been applied as the basis of biochemical detection in a number of situations. Two common approaches include transfer of small biomolecules across the 60 liquid-liquid interface facilitated by an ionophore, ${ }^{26,27}$ and ion transfer across the interface facilitated by biomolecules present in the aqueous phase and adsorbed at the interface. ${ }^{11,28}$ Scanlon et $a l .^{26}$ investigated the electroanalytical capabilities of microITIES with differential-pulse stripping voltammetry to detect the 65 transfer of small oligopeptides assisted by the dibenzo-18crown-6 (DB18C6) ether. This built on earlier work by Sawada and Osakai, ${ }^{27}$ who measured the transfer of hydrophobic peptides facilitated by DB18C6 and found good agreement between the half-wave potential and the peptide equivalent hydrophobicity 70 index. Recently, Méndez et al. ${ }^{28}$ simulated the adsorption of surface-active ions to liquid-liquid interfaces. They considered surfactant partitioning across the interface, whereby the adsorbed molecules could have portions in both the organic and aqueous phases. As discussed previously, the presence of hemoglobin at 75 the ITIES facilitated the transfer of hydrophobic anions to the aqueous phase. $^{11}$

There has been recent interest in label-free detection of biomolecules and biomolecular interactions. ${ }^{29,30}$ One approach to this has been electrochemical detection of adsorption (whether 80 specific or non-specific) based on changes in the conductance ${ }^{31-38}$ or impedance ${ }^{39}$ in response to biomolecules selectively adsorbing onto a functionalized working electrode. Many biomolecules of interest exist in ionized form, including nucleic acids, proteins, and many vitamins, biominerals and drugs, so electrochemistry at 85 the ITIES may be used for their detection. Additionally, the use of micron-sized pores to form microITIES results in a higher surface-to-volume ratio in the organic phase than would be present at ITIES formed at millimeter sized orifices. A high surface-to-volume ratio increases the likelihood of adsorption at 90 the pore wall without the need for mixing or convection, due to diffusive transport of the analyte to the surface. This leads to increased adsorption rates, so the influence of wall adsorption at 
micropores on voltammetric ion transfer behaviour may thus be more pronounced.

In the present paper, we explore the effects on ion transfer $\mathrm{CV}$ of analyte adsorption onto the walls of pores used to form 5 microITIES. This study is restricted to the case of a single microITIES formed at the mouth of a pore located within in a solid-state membrane. In such a system, analyte ions present on one side of the ITIES are transferred across the interface and adsorb onto the pore walls. This may be specific adsorption due 10 to the presence of bioreceptors on the pore wall or non-specific adsorption. The intention here was to determine whether such pore wall adsorption may impact the $\mathrm{CV}$ measurements at the ITIES and could form a basis for label-free electrochemical biosensing. Differences in the CVs for analytes that transfer 15 across the ITIES and adsorb to the walls, and those that transfer but do not adsorb, thus being available for back-transfer, may be used to identify the presence and concentration of given analytes, as well as to study adsorption/desorption processes. Finiteelement simulations of ion transfer across the interface between 20 an aqueous liquid and an organogel located within a micropore, combined with Langmuir adsorption to the pore wall, were used to identify system parameters that would be of interest in designing such a biosensor device. The effects of adsorption at different voltammetric scan rates and at smaller pore sizes were 25 of particular interest.

\section{Theory \& Methodology}

The interface formed at the micropore was modelled in two dimensions, taking advantage of the axial symmetry of the system to reduce the dimensionality. Ion-transfer kinetics were treated 30 with the Butler-Volmer model. ${ }^{40}$ Adsorption at the pore wall was described by Langmuir adsorption of a single analyte species. ${ }^{41-43}$ The model was evaluated over a range of pore geometries, adsorption site densities, and voltammetric scan rates. The effects of adsorption and desorption rate kinetics were also explored.

35 The computational domain is shown in Figure 1a and has been described previously. ${ }^{14}$ Due to the rotational symmetry of the system, a two-dimensional axi-symmetric model can be used.

The computational domain is divided into two subdomains, where subdomain 1 represents the organic phase, and subdomain 402 the aqueous phase. The two subdomains are connected by the micropore. The vertical origin $(x=0)$ is defined as the inlet to the pore from the aqueous side, and the radial origin $(r=0)$ is at the centre of the pore. The pore of length $l$ has dimensions $0 \leq x \leq x_{l}$, and radius $r_{a}$. The interface is located at $x=0$ and the reservoirs 45 extend to $x_{w}$ and $r_{w}$, and $x_{o}$ and $r_{o}$.

\section{Diffusion Model}

Diffusion of the analyte in domain $i$ is given by

$$
\frac{\partial c_{i}(x, r, t)}{\partial t}=D_{i} \nabla^{2} c_{i}(x, r, t)
$$

where $c$ is the concentration, $t$ is time, $D$ is the diffusion so coefficient, $i=1,2$ represent the organic and aqueous phases, respectively, and $\nabla^{2}$ is the Laplace operator for the axi-symmetric system,

$$
\nabla^{2}=\frac{\partial^{2}}{\partial x^{2}}+\frac{\partial^{2}}{\partial r^{2}}+\frac{1}{r} \frac{\partial}{\partial r}
$$

The initial conditions for the concentration are $c_{1}(x, r, 0)=0$ and ${ }_{55} c_{2}(x, r, 0)=c_{b}$, where $c_{b}$ is the bulk concentration of the analyte. The boundary equations at the liquid-liquid interface are (a) the Butler-Volmer type equation,

$$
\begin{aligned}
& \left.D_{1} \frac{\partial c_{1}(x, r, t)}{\partial x}\right|_{x=x_{s}} \\
& \quad=k^{0}\left[c_{1}\left(x_{s}, r, t\right) e^{-\alpha \frac{z_{z^{F}} F}{R T}\left(\Delta_{o}^{\prime \prime} \varphi-\Delta_{o}^{\prime \prime} \varphi^{\varphi^{\prime}}\right)}-c_{2}\left(x_{s}, r, t\right) e^{(1-\alpha) \frac{z_{i} F}{R T}\left(\Delta_{o}^{\prime \prime} \varphi-\Delta_{o}^{\prime \prime} \varphi^{0^{0}}\right)}\right]
\end{aligned}
$$

60 and (b) flux conservation,

$$
J_{0}=\left.D_{1} \frac{\partial c_{1}(x, r, t)}{\partial x}\right|_{x=x_{s}}=-\left.D_{2} \frac{\partial c_{2}(x, r, t)}{\partial x}\right|_{x=x_{s}}
$$

where $J_{0}$ is the mass flux and $x_{s}$ is the location of the ITIES along the $x$ axis $\left(x_{s}=0\right)$.. For $\mathrm{CV}$, the triangular potential waveform is ${ }^{13}$

$$
\begin{aligned}
\Delta_{o}^{w} \varphi=\Delta_{o}^{w} \varphi_{\text {init }} \\
+2 \frac{\Delta_{o}^{w} \varphi_{\text {swich }}-\Delta_{o}^{w} \varphi_{\text {init }}}{\pi} \\
\quad \times \sin ^{-1}\left[\sin \left(\frac{1}{2} \frac{\pi v t}{\Delta_{o}^{w} \varphi_{\text {swich }}-\Delta_{o}^{w} \varphi_{\text {init }}}\right)\right]
\end{aligned}
$$

${ }_{65}$ where $\Delta_{o}^{w} \varphi_{\text {init }}$ and $\Delta_{o}^{w} \varphi_{\text {swich }}$ are the initial and switching potentials, $v$ is the scan rate, and $t$ is time. The far-field boundary conditions were $c_{1}(x, r, t)=0$ and $c_{2}(x, r, t)=c_{b}$. In Equation (3) $k^{0}$ denotes the standard rate constant for ion transfer, $\alpha$ is the transfer coefficient and $\Delta_{o}^{w} \varphi^{0}$ is the formal ion transfer potential

70 The current was given by the integration of the surface flux normal to the interface,

$$
i=\left.2 \pi z_{i} F D_{2} \int_{0}^{r_{a}} r \frac{\partial c_{2}(x, r, t)}{\partial x}\right|_{x=x_{s}} d r
$$

where $r_{a}$ is the pore radius. The limiting current is $i_{\lim }=4 z_{i} F D_{2} c_{b} r_{a}$. Finally, the no-flux boundary condition 75 normal to the solid walls is $\partial c_{i} / \partial \mathbf{n}=0$, where $\mathbf{n}$ is a vector normal to the wall.

\section{Adsorption model}

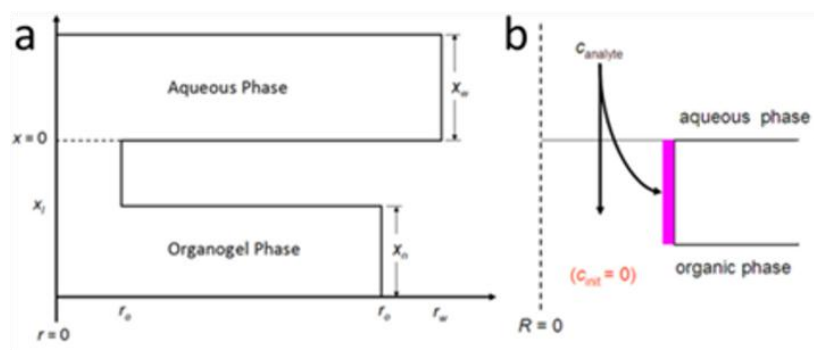

Fig. 1 (a) Simulation cell showing both phases and the geometric parameters of interest as described in the text. (b) Schematic representation of analyte adsorption onto the walls of a pore filled will 
organic liquid, following analyte transfer across the liquid-liquid interface.

Following $\mathrm{Hu}$ et $a .^{41}$, Langmuir-style adsorption behaviour was used for the pore wall. The binding reaction at the wall is

$5 A+B \underset{k_{d e s}}{\stackrel{k_{a d s}}{\rightleftarrows}} S$

where $A$ is the analyte species in phase $i$ with concentration $c_{i}, B$ represents the free binding sites with surface concentration $b, S$ are the bound analyte molecules with surface concentration $c_{s}$, and $k_{a d s}$ and $k_{d e s}$ are the adsorption and desorption rate constants, 10 respectively.

The reaction-diffusion equation for bound analytes, in terms of the solution concentration of analytes and the free bound sites, is ${ }^{42}$

$$
\frac{\partial c_{s}}{\partial t}=D_{s} \nabla^{2} c_{s}+k_{a d s} c_{i} b-k_{d e s} c_{s}
$$

15

where $D_{s}$ is the surface diffusivity of the bound complexes. We can write $b$ as the difference between the initial concentration of surface binding sites $b_{0}$ and the number of occupied sites $c_{s}$, so $b=b_{0}-c_{s}$. The reaction-diffusion equation, Equation 8, becomes

${ }_{20} \frac{\partial c_{s}}{\partial t}=D_{s} \nabla^{2} c_{s}+k_{a d s} c_{i}\left(b_{0}-c_{s}\right)-k_{d e s} c_{s}$

Equation (9) is the mass balance of surface-bound and solution-based analytes. The flux of the analyte normal to the reacting surface is given by ${ }^{41}$

$$
\mathbf{n} \cdot\left(-D_{i} \nabla c_{i}\right)=-k_{a d s} c_{i}\left(b_{0}-c_{s}\right)+k_{d e s} c_{s}
$$

25 which is the BC (boundary condition) applied to the solutionbased analyte at the adsorbing wall only. Equation 10 describes the adsorption and desorption of the analyte from the wall. To fully describe the reaction at the pore wall, a $\mathrm{BC}$ is required for the flux of the bound analyte normal to the surface ${ }^{41}$

${ }_{30} \mathbf{n} \cdot\left(-D_{s} \nabla c_{s}\right)=0$

Equation 11 is only applied to the wall within the pore. At all other walls in the system, the insulating boundary condition is used,

$$
\mathbf{n} \cdot\left(-D_{i} \nabla c_{i}\right)=0
$$

${ }_{35}$ Equations 1, 3, 4 and 9-12 are solved using appropriate system parameters. To minimize round-off errors and generalize the solutions, dimensionless variables were used. These are described in the Table S. 1 in the Supplementary Information, together with the dimensionless forms of the relevant equations.

\section{${ }_{40}$ Simulation parameters}

All simulations were carried out using COMSOL 3.5a equipped with the Chemical Engineering module. The simulation cell is shown in Figure 1. Two application modes were used to describe the system: a 2D axi-symmetric diffusion mode represented the
45 aqueous and organic phases, and adsorption was modelled using with COMSOL's 1D weak form boundary. A description of the weak form BC is given in the Supplementary Information. The simulations were performed using dimensionless variables, which are described in the Supplementary Information, but are 50 presented here in dimensional form. This was done to facilitate visualization of realistic systems. Three dimensionless dependent variables were used: $C_{1}, C_{2}$, and $C_{s}$, for the organic, aqueous, and surface concentrations.

A user-defined, problem-adapted mesh was used, with mesh 55 parameters chosen according to convergence tests. The maximum element size was 0.002 on the ITIES boundary and 0.01 at the adsorbing wall and at the points of the pore's inlet and outlet corners. Additionally, the mesh curvature factor on the ITIES was set to 0.1 . The mesh was further refined around the pore using the ${ }_{60}$ Mesh Selected command. For adsorption in the organic phase, a typical mesh was comprised of 70000 elements, with 147000 degrees of freedom. The UMFPACK linear solver was used, with an absolute tolerance of 0.000005 and a relative tolerance of 0.0001 .

${ }_{65}$ The parameters used for all simulations, unless otherwise indicated, were $c_{b}=10^{-7} \mathrm{~mol} \cdot \mathrm{cm}^{-3}, \quad D_{1}=9.86 \times 10^{-7} \mathrm{~cm}^{2} \cdot \mathrm{s}^{-1}$, $D_{2}=9.86 \times 10^{-6} \mathrm{~cm}^{2} \cdot \mathrm{s}^{-1}, v=10 \mathrm{mV} \cdot \mathrm{s}^{-1}, \Delta^{w} \varphi^{0}=0.55 \mathrm{~V}, \alpha=0.5$, $\varphi_{\text {init }}=0.2 \mathrm{~V}, \varphi_{\text {switch }}=1.1 \mathrm{~V}, k_{0}=0.5 \mathrm{~cm} \cdot \mathrm{s}^{-1}, k_{\text {ads }}=10^{8}$ $\mathrm{cm}^{3} \cdot \mathrm{mol}^{-1} \cdot \mathrm{s}^{-1}, k_{\text {des }}=0 \mathrm{~s}^{-1}$, and $b_{0}=10 \mathrm{pmol} \cdot \mathrm{cm}^{-2}$. These values 70 represent typical experimental parameters of common bioanalytical systems. ${ }^{41,42}$ The diffusivity in the organogel phase was $10 \%$ of that in the aqueous phase, and both were held constant for all the simulations. The decreased diffusion coefficient is due to the organogel phase used to stabilize the 75 interface at the micropore. ${ }^{14}$

A value of $D_{s}=9.86 \times 10^{-6} \mathrm{~cm}^{2} \cdot \mathrm{s}^{-1}$ was used for the surface diffusivity, which yields $\gamma_{s}=1$, in dimensionless variables (see Supplementary Information). This value is somewhat higher than the surface diffusivity used in Ref. 41, but is within the range of 80 surface diffusivity for a variety of inorganic and protein systems. ${ }^{44}$.

\section{Results \& Discussion}

In this study, we have considered the case where the pore is filled 85 with the organic phase and analyte ions are transferred across the interface from the aqueous phase into the organic phase, as shown schematically in Figure 1b. The analyte in the organic phase may then adsorb onto the pore wall. We refer to the adsorbing species as the analyte.

\section{${ }_{90}$ Validation of the simulation cell}

Using $r_{a}=10 \mu \mathrm{m}$ and $v=10 \mathrm{mV} \mathrm{s}^{-1}$, the maximal extensions of the computational domain were set to $x_{m}=4200 \mu \mathrm{m}$ and $r_{m}=420$ $\mu \mathrm{m}$ in the aqueous phase, and $x_{n}=1500 \mu \mathrm{m}$ and $r_{n}=350 \mu \mathrm{m}$ in the organogel phase. The dimensions were adjusted for different 95 values of the pore radius and scan rate. These values are greater than the $10 \sqrt{D_{i} t}$ suggested by Britz and Strutwolf ${ }^{45}$ and were determined to be sufficient to model the bulk solution conditions, via a comparison between the no-flux and constant (bulk) concentration far-field boundary conditions. 

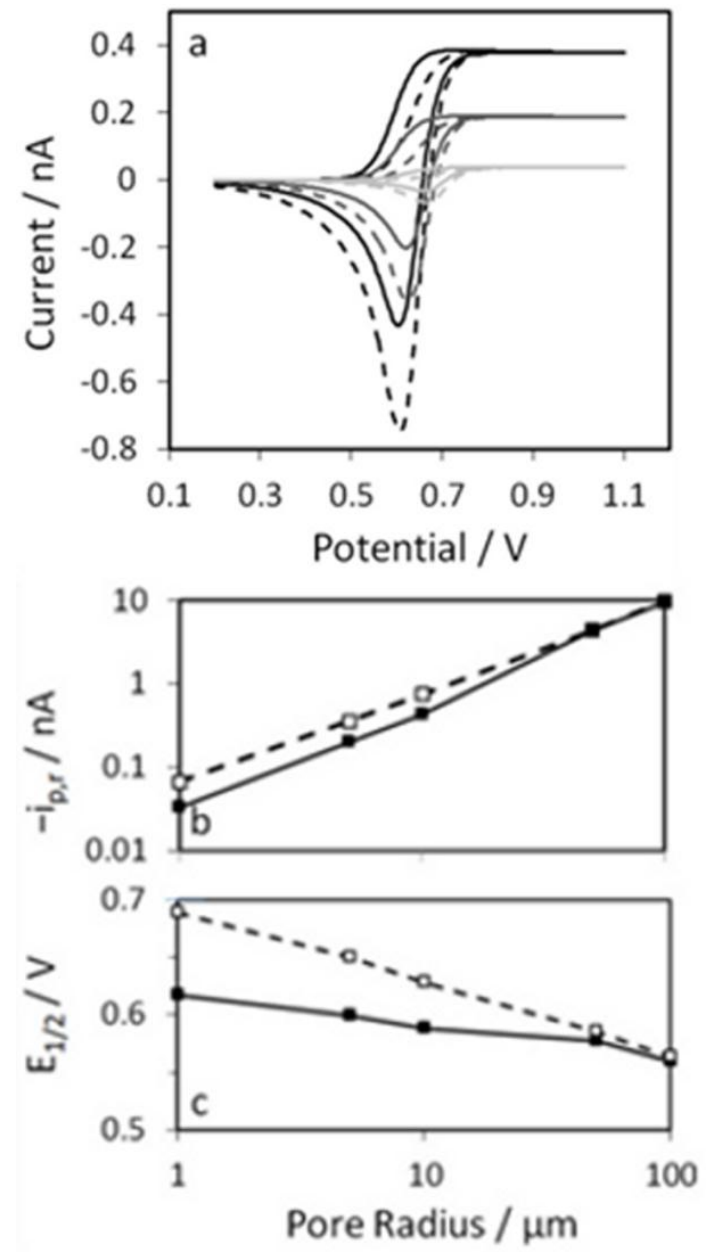

Fig. 2 Effects of pore radius on electrochemical signal with and without adsorption. (a) CV plots for $10-\mu \mathrm{m}$ (black), $5-\mu \mathrm{m}$ (dark grey), and $1-\mu \mathrm{m}$ (light grey) diameter pores with adsorption (solid line), compared to the

5 NoAds cases (dashed line). Effect of pore radius on (b) the reverse peak current and (c) the half-wave potential $\mathrm{E}_{1 / 2}$. In (b) and (c), the dashed lines show no adsorption and the solid lines are with adsorption. The lines are drawn as visualisation aids.

\section{Adsorption Simulations}

${ }_{10}$ Following validation of the simulation cells, a variety of adsorption parameters were compared. These included the pore radius $r_{a}$, adsorption and desorption rate constants $k_{a d s}$ and $k_{d e s}$, the density of adsorption sites $b_{0}$, and the scan rate. Of these, the scan rate was the most interesting as an analytical measure, since 15 it is easily varied experimentally.

\section{Effect of pore radius}

Simulations were performed for pore radii of 1,5 , and $10 \mu \mathrm{m}$. Figure 2a shows CVs for $r_{a}=1,5$ and $10 \mu \mathrm{m}$ for adsorption (Ads) and no adsorption (NoAds). Recall that all absolute 20 currents were calculated from the simulated dimensionless values using the limiting currents, $i_{\text {lim }}=4 z_{i} F D_{2} c_{b} r_{a}$,

The effect of adsorption was most apparent in the negative shift of the forward transfer wave for the $1 \mu \mathrm{m}$ interface. There was almost no difference in the height of the forward scan, 25 although there is a discernible shift to lower $E_{1 / 2}$ of the forward wave due to adsorption. The main indicators of adsorption are the decreases in the reverse peak (Fig. 2b) and the shift to lower halfwave potentials (Fig. 2c).
This can be attributed to two effects. The first involves edge 30 effects, which become important at smaller radii. The edge effect, however, is only responsible for an increase of flux density into the pore. What is more important is the increase in the surface-tovolume ratio within the pore as the radius decreases. Any effect occurring at the pore wall will be more pronounced, and 35 potentially more detectable, with increases in the surface-tovolume ratio.

For larger radii (50 and $100 \mu \mathrm{m}$ pores), adsorption has little effect on the reverse peak current and half-wave potential, such that the responses in Figs. $2 b$ and $2 c$ approach the NoAds ${ }_{40}$ behaviour. At these larger pore sizes, the inlet is sufficiently wide that any increase in the flux across the interface due to the adsorbing wall is outweighed by the linear bulk flux. The behaviour of the NoAds cases, as shown in Fig. $2 b$ and $2 c$, show the same linear dependence as was found by Josserand et al. ${ }^{12} \mathrm{We}$ ${ }_{45}$ feel that this qualitative agreement validates our computational approach.

\section{Effect of adsorption and desorption rate}

Figure 3 shows the effects due to changes in adsorption rate for the values of $k_{a d s}$ in the range $10^{3}$ to $10^{7} \mathrm{M}^{-1} \mathrm{~s}^{-1}$. The CVs in Fig.
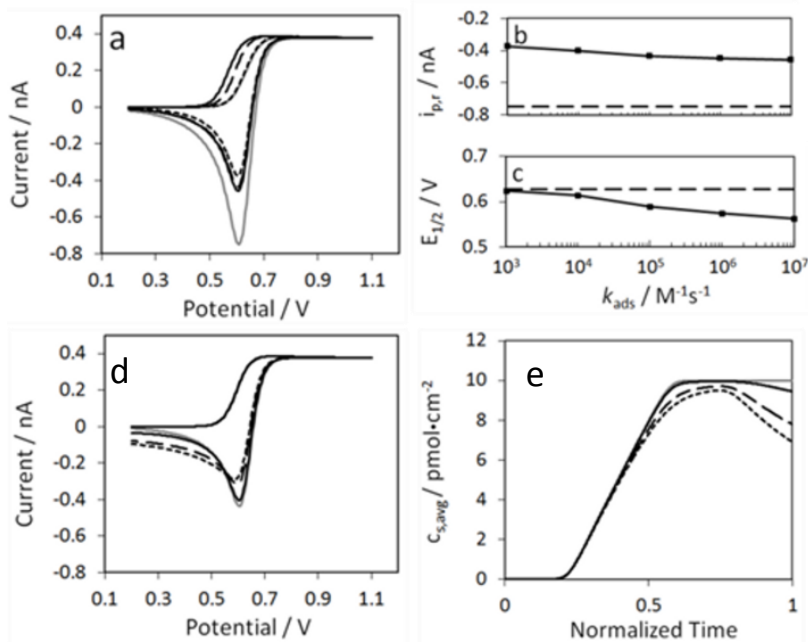

Fig. 3 Effects of adsorption (a-c) and desorption (d,e) rate constants on simulated responses. (a) Voltammogram showing the effects of adsorption for $k_{\text {ads }}=0$ (grey), $10^{7}(-), 10^{5}(---)$, and $10^{3}(--) \mathrm{M}^{-1} \mathrm{~s}$

${ }^{1}$. Effects of adsorption rate on the reverse peak current (b) and the half55 wave potential of the forward transfer process (c). In both (b) and (c), the constant dashed lines show the values for NoAds. The lines are drawn as visualisation aids. Voltammograms (d) and average surface concentration profiles (e) for different desorption rates $\left(k_{\text {des }}=0\right.$ (grey), $10^{-2}(-), 6 \times 10^{-2}$ $(---)$, and $10^{-1} \mathrm{~s}^{-1}(--)\left(k_{\text {ads }}\right.$ held constant at $\left.\left.10^{5} \mathrm{M}^{-1} \mathrm{~s}^{-1}\right)\right)$.

${ }_{60} 3 \mathrm{a}$ show the effect of various adsorption rates. The NoAds case is shown with dashed lines. The reverse peak height (Fig. 3b) became less negative by approximately $400 \mathrm{pA}$ as a result of adsorption. However, increasing $k_{a d s}$ from $10^{3}$ to $10^{7} \mathrm{M}^{-1} \mathrm{~s}^{-1}$ led to only gradual increases in the reverse peak current magnitude. ${ }_{65}$ Adsorption causes a shift in the half-wave potential (Fig. 3c) to lower potentials, and increases in $k_{a d s}$ resulted in further decreases.

The desorption rate constant $k_{\text {des }}$ was also varied between 0.001 and $0.1 \mathrm{~s}^{-1}$, with $k_{\text {ads }}$ held constant at $10^{5} \mathrm{M}^{-1} \mathrm{~s}^{-1}$. In the $\mathrm{CV}$ 70 plots (Fig. 3d), the forward peak remained the same, but the reverse peak broadened at higher values of $k_{\text {des. }}$. At higher values 
of the desorption constant $\left(k_{\text {des }}>10^{-2} \mathrm{~s}^{-1}\right)$, the current does not return to zero on the reverse scan due to the continued detachment of the desorbing species. Fig. 3e shows mean surface concentration profiles (averaged over the length of the wall) for 5 the desorption rates simulated over the course of the simulations (normalized to the total simulation times). As expected, higher desorption prevents the surface from becoming fully saturated.

\section{Effect of adsorption site density}

The density of adsorption sites was varied between $b_{0}=1$ $10 \mathrm{pmol} \cdot \mathrm{cm}^{-2}$ and $b_{0}=15 \mathrm{pmol} \cdot \mathrm{cm}^{-2}$, with the results shown in Figure 4. Fig. 4a shows CVs for the NoAds (dashed line) and Ads (solid lines) cases at various adsorption site densities. Figs. $4 \mathrm{~b}$ and $4 \mathrm{c}$ show the changes in $i_{p, r}$ and $\mathrm{E}_{1 / 2}$ with adsorption site density, respectively. The reverse peak current became smaller as $b_{0}$ 15 increased, as more ions were captured on the wall and could therefore not return to the aqueous phase on the reverse sweep. The decrease in $E_{1 / 2}$ with increasing site density is due to analyte transfers across the ITIES becoming easier with adsorption on the pore wall as an additional driving force. Plots of the CVs and 20 average surface concentrations for $b_{0}$ are shown in the Supplementary Information (Figure S.2).
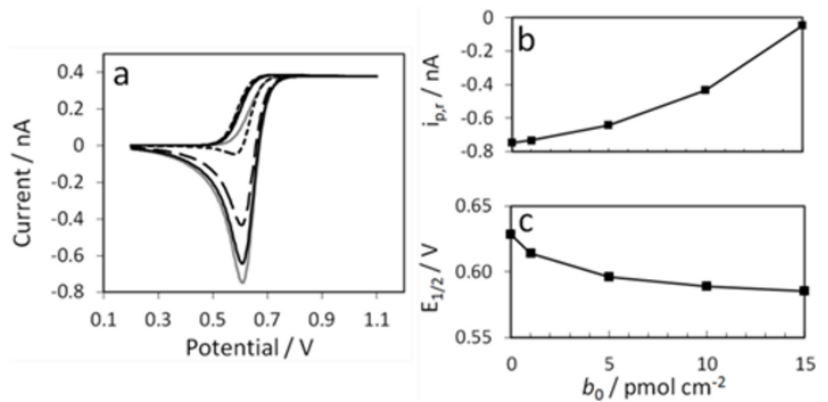

Fig. 4 Influence of surface concentration $b_{0}$ on the simulated CV response: (a) for $b_{0}=0$ (grey), 5 (-), $10(---)$, and $15(--)$ $25 \mathrm{pmol} \mathrm{cm} \mathrm{cm}^{-2}$. The effects in terms of the reverse peak currents (b) and the half-wave potential (c) are shown.

\section{Effect of scan rate}

Finally, the electrochemical behaviour was explored as a function of the scan rate. This is perhaps the most interesting scenario, 30 since this parameter is most easily varied experimentally. Five scan rates were simulated, 5, 10, 15, 50, and $100 \mathrm{mV} / \mathrm{s}$. Figures 5a and 5b show CVs for $v=10$ and $v=15 \mathrm{mV} \cdot \mathrm{s}^{-1}$, respectively, for the NoAds (dashed lines) and Ads (solid lines) cases. Interestingly, the scan rate presents the most promising metric for 35 adsorption detection, since the changes are so pronounced if adsorption is present. Notably, the reverse peak current became smaller at higher scan rates with adsorption, whereas it became larger with no adsorption. Also, at scan rates above $15 \mathrm{mV} \cdot \mathrm{s}^{-1}$, there was no reverse peak in the presence of adsorption. This is 40 illustrated in Figs. $5 \mathrm{c}$ and $5 \mathrm{~d}$ for $i_{p, r}$ and $\mathrm{E}_{1 / 2}$, respectively, over all simulated scan rates. The behaviour of the reverse peak current in the NoAds case is qualitatively similar to that found by Josserand et $a l .{ }^{12}$ at lower scan rates. Simulated CVs for the various scan rates are shown in the Supplementary Information (Figure S3).

45 As expected, there is no current peak on the forward scan for the NoAds case (dashed line in Fig. 5a), whereas a small peak appears for the Ads case. This is due to the aforementioned effect of the adsorbing wall acting as an attractive element for the analyte. As the analyte initially crossed the interface in response
50 to the increase in potential, it was immediately adsorbed to the pore wall. This temporarily decreased its concentration in the vicinity of the wall, increasing the concentration gradient and shifting the forward wave to a lower potential for the adsorbing wall case, across all scan-rates.

55 The behaviour of the reverse peak current for the NoAds case is also as expected - the reverse peak height increases with the scan rate. However, it is not linear because, at higher scan rates, the analyte does not fill the pore. In the Ads case, however, the peak height in fact decreases with increasing scan rate. Because 60 the diffusion layer did not penetrate all the way into the pore, a larger fraction of the transferred analyte was adsorbed, so less was available for back transfer to the aqueous phase. This effect was amplified as the scan rate increased, such that, for the simulated scan rates above $15 \mathrm{mV} \mathrm{s}^{-1}$, all the analyte was 65 adsorbed by the wall. It is a surface effect, so may be more pronounced for ITIES formed within nanoscale pores, ${ }^{21}$ where the surface-to-volume ratio is much larger.

The halfwave potential $\mathrm{E}_{1 / 2}$ shifted to a lower potential upon adsorption, due to an increased concentration gradient caused by 70 the attractive wall. The decrease in $\mathrm{E}_{1 / 2}$ in the absence of wall adsorption is explained as follows. The thickness of the diffusion zone established in the organic phase is already lower than that in
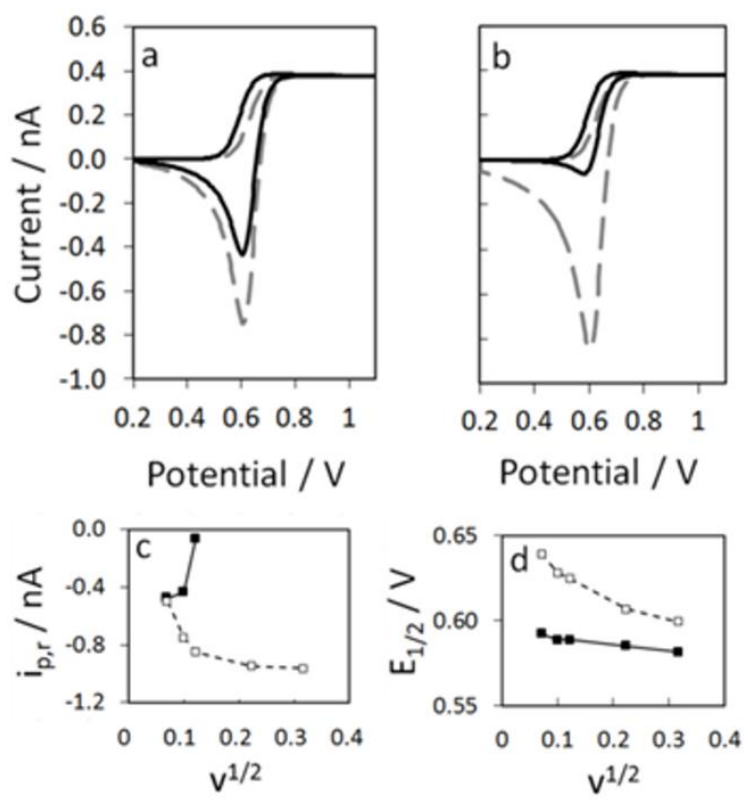

Fig. 5 Effects of scan rate on CV without and with pore wall adsorption, 75 for NoAds (dashed line) and Ads (solid lines), at $v=10$ (a) and 15 (b) $\mathrm{mV} \mathrm{s}^{-1}$. Comparison of the half-wave potential (c) and the reverse peak current (d) over a range of scan rates $\left(5\right.$ to $100 \mathrm{mV} \mathrm{s}^{-1}$ ) for NoAds (dashed line) and Ads (solid line). For the reverse peak current (c), no points are plotted for scan rates above $15 \mathrm{mV} \mathrm{s}^{-1}$ for the Ads case, as there was no 80 reverse peak.

the aqueous phase, due to the lower diffusivity in this phase $\left(D_{\text {org }}=0.1 D_{a q}\right)$. The thickness is further decreased as the diffusion time is decreased (as the scan rate is increased).

Therefore, with decreasing time of transfer into the organic 85 phase, the ions are located closer to the interface on the organic side and more readily available for back transfer during the reverse potential scan. This leads to a decrease of $E_{1 / 2}$ with increasing scan rate, if no effect other than diffusion is 
considered.

In the case of pore wall adsorption, the half-wave potential for the forward scan shifts to more negative potentials for the reasons discussed above, offsetting the effect on $E_{1 / 2}$ caused by the 5 decrease in diffusion layer thickness with increasing scan rate. The half-wave potential presents a good analytical measure for adsorption detection.

\section{Opportunities for biosensing}

The situations illustrated above demonstrate the potential 10 application of liquid|liquid electrochemistry for the detection of biomolecular adsorption, as well as some challenges which may be encountered. For adsorption on pore walls in the organic phase, the limiting current $i_{\text {lim }}=4 z_{i} F D_{2} c_{b u l k} r_{a}$, on the order of $40 \mathrm{pA}$ for a single $20-\mu \mathrm{m}$ diameter interface is sufficiently high 15 for detection, for bulk concentrations of $100 \mu \mathrm{M}$. Signal amplification could be achieved through the use of arrays of microinterfaces ${ }^{15,20}$, although care must be taken when designing such arrays to avoid overlapping diffusion fields between adjacent interfaces.

20 However, there are two challenges for using this configuration in biosensing: i) delivery of biologically interesting analytes in an organogel and ii) biological activity of the surface-bound moiety. The first issue would be a concern in the case of some oligopeptides or nucleotides, where the molecular activity may be

25 compromised in the organic phase, and would not recover in the aqueous phase. However, for small ions, and possibly even short oligonucleotides, this will not be a serious concern. The second issue could present a challenge since a lack of activity in the immobilized species would compromise biosensor function. 30 However, the study of enzyme activity in organic solvents is itself an interesting field, ${ }^{46-48}$ to which this technique could be applied. Such a configuration might also be applied to the measurement of membrane proteins interacting with ionized species.

\section{${ }_{35}$ Conclusions}

Using computational simulation, we have investigated the influence of pore wall adsorption on ion transfer electrochemistry at immiscible liquid-liquid interfaces formed at a micropore. The main observations from this study are that pore wall adsorption 40 following transfer from aqueous to organic phases results in (1) a shift of the half-wave potential for the water-organic transfer process to lower potentials and (2) a decrease in the magnitude of the reverse scan CV current. These are due to the pore wall adsorption process providing an additional driving force to bring 45 the ion across the interface and to the adsorbed material being unavailable for back transfer during the course of the reverse CV scan. The effects of adsorption become most notable at smaller pore diameters, where the change in the half-wave potential is most pronounced. The disappearance of the reverse peak at 50 higher scan rates and higher surface concentrations, as well as the changes in the half-wave potential and reverse peak current, could serve as indicators of pore wall adsorption. This is especially the case since the scan rate is easily varied experimentally. Detection could be improved by examining a 55 combination of metrics and factors, including changes in the halfwave potential and the reverse peak current with scan rate.
Effects due to adsorption and desorption rates, and binding site density were also presented.

While the strategy has not been experimentally verified, this 60 work reveals the promise of such an arrangement for adsorption and other surface interaction studies. This method could be applied to a number of bio-detection strategies. One such class of systems might involve the measurement of enzyme-cofactor binding constants. Another possible application might involve the 65 measurement of adhesion kinetics for aqueous-phase small molecules to surface-bound receptors in the organic phase, following transfer from the aqueous phase.

Additionally, such a system would not be limited to biochemical adsorption studies. The Langmuir adsorption 70 isotherm is a versatile model, and can be applied as a first approximation for most surface-catalysed chemical reactions. Furthermore, more complex adsorption models could be employed.

\section{Acknowledgements}

75 The authors are grateful to Science Foundation Ireland (grant number 07/IN.1/B967) and the Irish Research Council for Science, Engineering and Technology (Government of Ireland Postdoctoral Fellowship to JSE) for support of this research.

\section{Notes and references}

${ }_{80}{ }^{a}$ Tyndall National Institute, Lee Maltings, University College Cork, Cork, Ireland.

${ }^{b}$ Nanochemistry Research Institute, Department of Chemistry, Curtin University, GPO Box U1987, Perth, WA 6845, Australia. Fax: +61-89266-4699; Tel: Tel: +61-8-9266-9735; E-mail:

85 D.Arrigan@curtin.edu.au.

$\dagger$ Electronic Supplementary Information (ESI) available: Modeling equations in terms of the dimensionless variables, weak boundary condition for surface adsorption, $\mathrm{CV}$ plots for various adsorption rate constants $k_{a d s}, \mathrm{CV}$ plots and surface concentration profiles for $b_{0}$, and $\mathrm{CV}$ 90 plots for various scan rates. See DOI: $10.1039 / \mathrm{b} 000000 \mathrm{x} /$

* Present Address: Mechanical and Industrial Engineering, University of Toronto, Toronto, Canada.

II Present Address: Institut für Organische Chemie, Fachbereich Chemie, Universität Tübingen, Germany.

95

1. Z. Samec, Pure and Applied Chemistry, 2004, 76, 2147.

2. F. Reymond, D. Fermin, H. J. Lee and H. H. Girault, Electrochimica Acta, 2000, 45, 2647-2662.

3. B. Liu and M. V. Mirkin, Electroanalysis, 2000, 12, 1433-1446.

100 4. H. L. T. Ho and R. A. W. Dryfe, Langmuir, 2009, 25, 12757-12765.

5. F. O. Laforge, P. Sun and M. V. Mirkin, Journal of the American Chemical Society, 2006, 128, 15019-15025.

6. Y. H. Shao, M. V. Mirkin and J. F. Rusling, Journal of Physical Chemistry B, 1997, 101, 3202-3208.

105 7. Y. H. Shao and M. V. Mirkin, Journal of the American Chemical Society, 1997, 119, 8103-8104.

8. Y. Yuan and Y. H. Shao, Journal of Physical Chemistry B, 2002, 106, 7809-7814.

9. F. Kivlehan, Y. H. Lanyon and D. W. M. Arrigan, Langmuir, 2008, $110 \quad 24,9876-9882$.

10. D. G. Georganopoulou, D. J. Caruana, J. Strutwolf and D. E. Williams, Faraday Discussions, 2000, 116, 109-118. 
11. G. Herzog, V. Kam and D. W. M. Arrigan, Electrochimica Acta, 2008, 53, 7204-7209.

12. J. Josserand, J. Morandini, H. J. Lee, R. Ferrigno and H. H. Girault, Journal of Electroanalytical Chemistry, 1999, 468, 42-52.

5 13. P. J. Rodgers and S. Amemiya, Analytical Chemistry, 2007, 79, 9276-9285.

14. J. Strutwolf, M. D. Scanlon and D. W. M. Arrigan, Analyst, 2009, 134, 148-158.

15. R. Zazpe, C. Hibert, J. O'Brien, Y. H. Lanyon and D. W. M. Arrigan, $10 \quad$ Lab on a Chip, 2007, 7, 1732-1737.

16. Q. Li, S. B. Xie, Z. W. Liang, X. Meng, S. J. Liu, H. H. Girault and Y. H. Shao, Angewandte Chemie-International Edition, 2009, 48, 8010-8013.

17. P. J. Rodgers, S. Amemiya, Y. X. Wang and M. V. Mirkin, 15 Analytical Chemistry, 2010, 82, 84-90.

18. C. X. Cai, Y. H. Tong and M. V. Mirkin, Journal of Physical Chemistry B, 2004, 108, 17872-17878.

19. Y. X. Wang, J. Velmurugan, M. V. Mirkin, P. J. Rodgers, J. Kim and S. Amemiya, Analytical Chemistry, 2010, 82, 77-83.

20 20. S. Wilke, M. D. Osborne and H. H. Girault, Journal of Electroanalytical Chemistry, 1997, 436, 53-64.

21. M. D. Scanlon, J. Strutwolf, A. Blake, D. Iacopino, A. J. Quinn and D. W. M. Arrigan, Analytical Chemistry, 2010, 82, 6115-6123.

22. C. Beriet, R. Ferrigno and H. H. Girault, Journal of Electroanalytical

25 Chemistry, 2000, 486, 56-64.

23. T. J. Davies and R. G. Compton, Journal of Electroanalytical Chemistry, 2005, 585, 63-82.

24. H. J. Lee, C. Beriet, R. Ferrigno and H. H. Girault, Journal of Electroanalytical Chemistry, 2001, 502, 138-145.

30 25. N. Godino, X. Borrisè̀ $\square$, F. X. Munl̀ $f$ oz, F. J. del Campo and R. G. Compton, The Journal of Physical Chemistry C, 2009, 113, 1111911125 .

26. M. D. Scanlon, G. Herzog and D. W. M. Arrigan, Analytical Chemistry, 2008, 80, 5743-5749.

35 27. S. Sawada and T. Osakai, Physical Chemistry Chemical Physics, 1999, 1, 4819-4825.

28. M. A. Mendez, B. Su and H. H. Girault, Journal of Electroanalytical Chemistry, 2009, 634, 82-89.

29. I. Vlassiouk, T. R. Kozel and Z. S. Siwy, Journal of the American

40 Chemical Society, 2009, 131, 8211-8220.

30. T. D. Lazzara, I. Mey, C. Steinem and A. Janshoff, Analytical Chemistry, 2011, 83, 5624-5630.

31. B. Zhang, M. Wood and H. Lee, Analytical Chemistry, 2009, 81, 5541-5548.

45 32. R. M. M. Smeets, U. F. Keyser, D. Krapf, M. Y. Wu, N. H. Dekker and C. Dekker, Nano Letters, 2006, 6, 89-95.

33. D. Fologea, M. Gershow, B. Ledden, D. S. McNabb, J. A. Golovchenko and J. Li, Nano Letters, 2005, 5, 1905-1909.

34. J. L. Li, M. Gershow, D. Stein, E. Brandin and J. A. Golovchenko, $50 \quad$ Nature Materials, 2003, 2, 611-615.

35. H. Chang, F. Kosari, G. Andreadakis, M. A. Alam, G. Vasmatzis and R. Bashir, Nano Letters, 2004, 4, 1551-1556.

36. S. M. Iqbal, D. Akin and R. Bashir, Nat Nano, 2007, 2, 243-248.

37. R. Fan, R. Karnik, M. Yue, D. Li, A. Majumdar and P. Yang, Nano

55 Letters, 2005, 5, 1633-1637.

38. O. A. Saleh and L. L. Sohn, Nano Letters, 2003, 3, 37-38.
39. I. Vlassiouk, P. Takmakov and S. Smirnov, Langmuir, 2005, 21, 4776-4778.

40. A. J. Bard and L. R. Faulkner, Electrochemical Methods :

60 Fundamentals and Applications, 2nd edn., John Wiley, New York, 2001.

41. G. Q. Hu, Y. L. Gao and D. Q. Li, Biosensors \& Bioelectronics, 2007, 22, 1403-1409.

42. T. Gervais and K. F. Jensen, Chemical Engineering Science, 2006,

$65 \quad 61,1102-1121$.

43. R. Winz, A. de los Rios Gonzalez, E. von Lieres, M. Schmittel and W. Wiechert, Proceedings of the Comsol Users Conference, Grenoble, France, 2007.

44. V. Chan, D. J. Graves, P. Fortina and S. E. McKenzie, Langmuir, 1997, 13, 320-329.

45. D. Britz and J. Strutwolf, Electrochimica Acta, 2006, 52, 33-41.

46. A. Zaks and A. M. Klibanov, Proceedings of the National Academy of Sciences of the United States of America, 1985, 82, 3192-3196.

47. A. M. Klibanov, Trends in Biochemical Sciences, 1989, 14, 141-144. 75 48. M. N. Gupta, European Journal of Biochemistry, 1992, 203, 25-32. 